\title{
Network Architecture of Wireless Underground Sensor Networks for Oil and Gas Pipeline Monitoring
}

\author{
Huaping $\mathrm{Yu}^{1, \mathrm{a}^{*}, \text { Lan Huang }}{ }^{1, \mathrm{~b}}$ and Mei Guo ${ }^{2, \mathrm{c}}$ \\ ${ }^{1}$ College of Computer Science, Yangtze University, Jingzhou 434023, China \\ ${ }^{2}$ Department of Science and Technology, Yangtze University, \\ Jingzhou 434023, China \\ ayhpjz@126.com, ${ }^{b}$ lhuang@126.com, ${ }^{c}$ guomei@yangtzeu.edu.cn
}

\begin{abstract}
Oil and gas pipelines are the infrastructure of national economic development. A flexible and energy efficient network architecture is the fundament of wireless underground sensor networks (WUSN) for oil and gas pipeline systems. This paper firstly analyzes the underground wireless channel characteristics of the electromagnetic wave (EM), energy consumption dissipation of sensor nodes with EM, changing rule of the path loss factors of EM in soil and points out the energy efficiency and energy balance problem of WUSN for underground oil and gas pipeline monitoring. Secondly, the typical three-layers network architecture of WUSN for oil and gas pipelines monitoring is introduced, and give a practical data collection operation step. Thirdly, some effective improvement strategies for the three-layers network architecture to improve energy performance are proposed, which include (1) magnetic induction waveguide technique, (2) data collection strategies through aboveground and underground mobile nodes, (3) the energy replenishment strategies of sensor nodes, (4) line non-uniform and uniform sensor nodes deployment and scheduling strategies. At same time, simulation analysis and discussion of above all strategies are developed, the analysis result shows that the proposed strategies can effectively improve the energy performance and prolong the network life span.
\end{abstract}

Keywords: oil and gas pipeline, soil environment, energy performance, network architecture, node deployment and scheduling

\section{Introduction}

Wireless underground sensor network (WUSN) is a main technical method to solve the problem of various kinds of information monitoring in near-surface underground soil environment [1], which consists of a large number of resourcelimited sensor nodes such as limited energy resource, limited storage capacity and limited computation ability. Each sensor node is typically equipped with different types of sensors, computational units, storage devices and communication modules, sensor nodes are deployed with random or deterministic way in the underground monitoring area, which can automatically sense, process, and transmit all kind of monitored data, without any human intervention.

Oil and gas pipelines are vital infrastructure to national economy, which is the most economical way to transport crude oil, natural gas and chemical products due to its lower costs, higher capacity and better consistency than alternative transporting methods such as railroad and highway [2]. However, managing oil and gas pipelines is challenging, especially with the rapid growth in the length of the oil and gas pipelines. Successful oil and gas pipeline management must monitor leak,

${ }^{*}$ Corresponding Author 
pressure, flow, corrosion, pollution in surrounding environment, and many other factors that affect the safety of the oil and gas pipelines and thus transportation efficiency. It is obvious that WUSN has many advantages to construct oil and gas pipeline monitoring systems in the terms of the cost performance, flexible and control efficiency, which can effectively improve the level of management and economic and social benefits. For example, WUSNs can monitor the pipeline information and estimate pipeline state unremittingly without manual intervention and work at the night and abominable weather. [2-3].

The network architecture of WUSN, sensor node deployment and scheduling are the fundamental problems of WUSN for oil and gas pipeline monitoring. Recently, researchers have proposed pipeline monitoring system with WUSN technology [36]. Literature [3] proposes an efficient pipeline state information collection algorithm based on sensor node line deployment strategy and data fusion strategy, the result of simulation and analysis shows that the proposed algorithm can remarkably improve the network performances on delay and energy, ensure the urgency data to be propagated effectively and prolong the network life span. Literature [4] discussed the feasibility and the application prospect of WSN/WUSN to monitor pipelines. Literature [5] studied the spatial structure of oil and gas pipelines and introduced the three layers system structure of WUSN for oil and gas pipelines monitoring, and proposed an optimal deployment strategy in XY plane and $\mathrm{XZ}$ plane which were projected from three-dimensional oil and gas pipeline structure. Literature [6] introduced a magnetic induction (MI)-based wireless sensor network for underground pipeline monitoring (MISE-PIPE). But, the above network architectures of WUSN lack of flexibility for oil and gas pipeline monitoring, which are not designed on the whole and not fully considering the characteristics of the oil and gas pipeline monitoring. Therefore, the energy efficiency and energy balance of WUSN have a large room to improve.

The remainder of this paper is organized as follows. In Section II, the features of underground wireless channel, energy consumption model and pipeline monitoring requirements are analyzed. Then in Section III, typical three tires network architecture of WUSN for oil and gas pipelines monitoring is discussed. In Section IV, four improvement strategies are proposed to set up a flexible and energy efficient network architecture of WUSN for oil and gas monitoring. Finally, the paper is concluded in Section V.

\section{Underground Wireless Channel}

\subsection{Underground wireless channel characteristics of electromagnetic wave.}

At present, the communication technology of the wireless sensor network (WSN) is based on the electromagnetic wave (EM). But, the communication link in nearsurface underground soil has obvious space-variant features. Channel capacity is vulnerably affected by many factors such as the work frequency (denoted as F) of electromagnetic waves, soil water content (denoted as SWC) and space-variant features of channel [7]. Specifically, path loss (denoted as LEM (d)) of near-surface underground soil environment can be expressed as,

$$
L_{E M}(d)=6.4+20 \log (d)+20 \log (\beta)+8.69 \alpha d,
$$

Where $\mathrm{d}$ is the distance between the adjacent sensor nodes, the unit is meter; $\alpha$ is the attenuation coefficient, the unit is $1 / \mathrm{m}$; and $\beta$ is the phase shift coefficient, the unit is radian $/ \mathrm{m}$. the experiment and simulation results show as follow:

The 300-500 MHz is feasible work frequency for wireless communication in near-surface underground soil environment using EM waves. The greater the working frequency, the greater the path loss; the greater soil volumetric water 
content, the greater the path loss; the path loss in near-surface underground soil environment is more than four to six times in the free space (such as in air). The simulation analysis results are shown in Figure 1.

The maximum effective transmission distance between the adjacent sensor nodes is no more than 4 meters with $433 \mathrm{MHz}$ in near-surface underground soil environment. The greater the transmission distance, the greater the path loss; As shown in Figure 2, it is shown that in the $300-500 \mathrm{MHz}$ frequency band, the path loss can be limited to a degree $(<=100 \mathrm{~dB})$ supporting feasible communication in the near-surface underground soil environment, of which the maximum transmission distance (Rc) between the sensor nodes is not more than $4 \mathrm{~m}$, and effective distance perception of sensor nodes (Rs) is about the same or even lower than Rc [7]. But the effective communication distance of sensor nodes in air is more than $80 \mathrm{~m}$, which is greater than the effective distance perception, namely the Rc 》 Rs. and when the Rc 》Rs, the node deployment algorithm can guarantee network connectivity if the algorithm can seamlessly cover monitoring area. Therefore, network connectivity is the important factors which need to consider in designing the architecture of WUSN for oil and gas pipelines monitoring.



Figure 1. Path Loss Versus Operating Frequency and Soil Water Content (SWC)

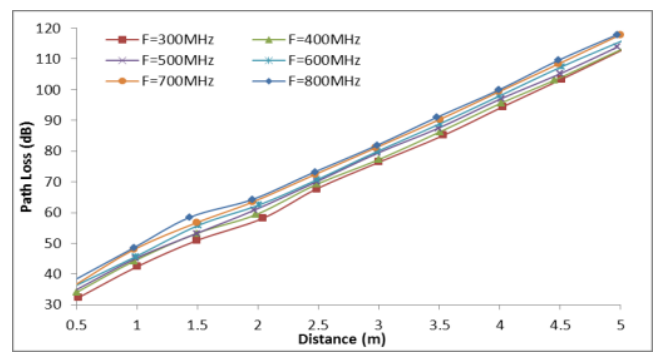

Figure 2. Path Loss Versus Operating Frequency and Internode Distance

\subsection{Energy Consumption Model of EM}

Radio energy dissipation model

Energy resource of sensor node is very limited. Its energy consumption is related to the life cycle of the network. The classic sensor node energy consumption model is First Order Radio Frequency model which was putted forward by the Heinzelman [8] and was as shown in Figure 3 and the energy consumption formula is given in (2).

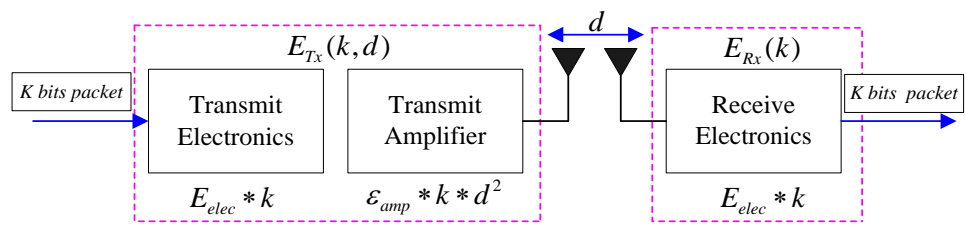

Figure 3. Radio Energy Dissipation Model

$$
\left\{\begin{array}{c}
E_{T x}(k, d)=k E_{\text {elec }}+k \varepsilon_{\mathrm{amp}} d^{\gamma} \\
E_{R x}(k, d)=k E_{\text {elec }}
\end{array},\right.
$$

Where $\mathrm{k}$ is the transmission data quantities, the unit is bits; $\mathrm{d}$ is the one hop transmission distance, the unit is meter; $\gamma$ is the path loss factor, which is $2 \sim 4$ in air medium; Eelec is the circuit RF loss of transmitter and receiver at work, Eelec $=50 \mathrm{~nJ} / \mathrm{bit}$, Eamp is the amplifier loss of transmitter, eamp $=0.0013 \mathrm{pJ} / \mathrm{bit} / \mathrm{m} 2$. It is obvious that transmission data quantities $(\mathrm{k})$, path attenuation factor $(\gamma)$ and 
transmission distance (d) are the key factors that influencing the energy consumption of sensor nodes. The circuit RF loss of transmitter and receiver (Eelec) and amplifier loss of transmitter (camp) is relatively stable. Specifically, parameter $\mathrm{k}$ is related to the specific mission requirements and it can be reduced by data fusion and prediction technologies and data collecting strategies; parameter $\mathrm{d}$ can be optimized with the sensor nodes partition strategies; while parameter $\gamma$ is easily affected by work environment.

Path loss factors in soil

According to the literature [8], the path loss factors in air (marked as yair) is 2 4, but the path loss factors in soil (marked as $\gamma$ soil) is far greater than the value of 2 4 and $\gamma$ soil is 4 5.5 times of yair from figure 1. The parameter $\gamma$ soil is easily affected by buried depth of sensor node, operating frequency, soil water content and other factors, which are shown in Figure 4. In Figure 4, the parameters values are set as follows: soil water content is $5 \%$, soil composition includes $50 \%$ sand, $15 \%$ clay and $35 \%$ salt. The path loss factors $\gamma$ soil is shown as a function burial depth for various operating frequency. It can be observed that an optimum burial depth exists such that the path attenuation factor is minimized for a particular operating frequency, and the higher the operating frequency, the smaller the optimal burial depth of sensor nodes. Therefore, the range of $\gamma$ soil is 10 16 [9].

A typical sensor node energy consumption distribution is shown in Figure 5 [10].

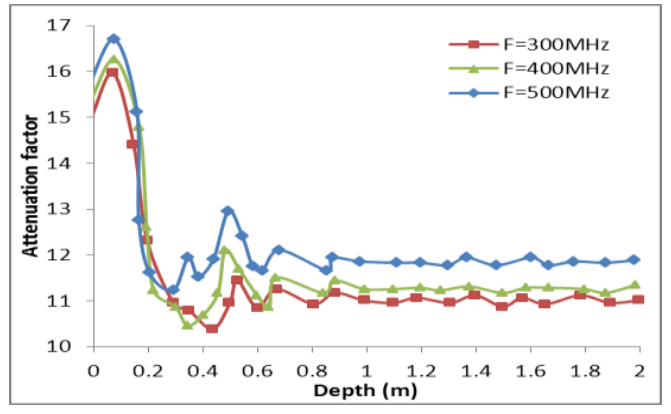

Figure 4. The Path Loss Factors in
Soil (ysoil) Versus Burial Depth
for Different Operating Frequencies

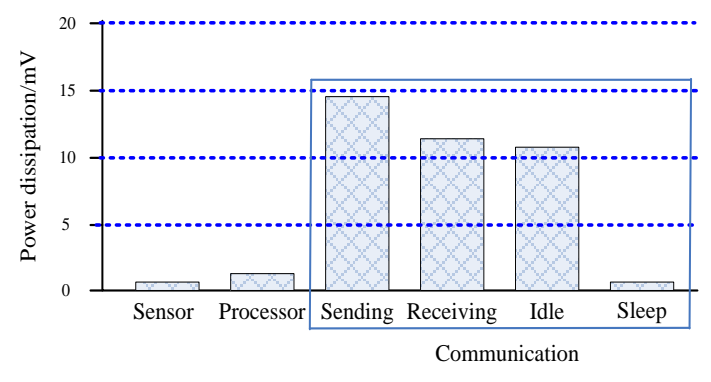

Figure 5. Energy Consumption Distribution of Sensor Nodes

Energy consumption distribution of sensor nodes.

As can be seen from figure 5, the energy consumption of the sensor node is mainly from the wireless communication module, the microprocessor module and sensor module. And transmitting information of sensor node needs more electricity than performing calculations and sensing data, the energy of transmitting 1 bit data is enough to perform 3000 computation instructions, so almost all the energy of the nodes will consume on the wireless communication module [10]. Therefore, reducing the energy consumption of WUSN relies heavily on reducing the number of communication and the amount of transmission data.

Oil pipelines are made from steel or plastic tubes with inner diameter typically from 100 to $1,200 \mathrm{~mm}$. Most pipelines are typically buried at a depth of about 0.91 to $1.8 \mathrm{~m}$. The oil is kept in motion by pump stations along the pipeline, and usually flows at speed of about 1 to 6 meters per second. For natural gas, pipelines are constructed of carbon steel and vary in size from 51 to $1,500 \mathrm{~mm}$ in diameter, depending on the type of pipeline. The gas is pressurized by compressor stations [2].

On the one hand, oil and gas pipeline are usually deployed in near-surface underground soil, which have much more energy consumption of wireless underground channel compared with the energy consumption in free space. On the 
other hand, oil and gas pipeline are typical long linear structure, which leads to the problem of grievous non-uniformity energy consumption among the long lineardistributed sensor nodes. These problems subsequently reduce the lifetime of WUSN. From the point of view of data collection requirements, oil and gas pipeline monitoring information has the characteristics of the temporal and spatial correlations. Neighboring sensor nodes report similar (but not equal) data values which have high degree of correlations, which is spatial correlation. And the temporal correlation is observed that successive sensed parameter values by the same sensor node are found to be identical and varies slowly except in the case of unexpected events. The spatial and temporal correlations of the oil and gas pipeline monitoring information can prove that data fusion and forecasting are necessary and feasible method to improve system performance through decreasing the transmission frequency and transmission data volume. Moreover, network architecture of WUSN is the foundation of data fusion and forecasting. Therefore, designing flexible and energy efficient network architecture is a key to improve network performance, especially for energy performance.

\section{Network Architecture of WUSN for Oil and Gas Pipelines Monitoring}

Sensor nodes that deployed in the oil and gas pipeline system is mainly divided into two kinds: one kind is sensor nodes that deployed outside of pipe wall, mainly used to judge the pipeline leakage and detect soil parameters such as soil moisture, soil temperature and hydrocarbon contents. Another kind is sensor nodes that deployed inside of pipe wall, mainly including acoustic detection equipment, pressure sensor, flow sensor and temperature sensor, which used to detect pipeline state and fluid flow information. In order to ensure monitoring performance of oil and gas pipeline system, we use the typical three layers system structure, which is shown in Figure 6 [6].

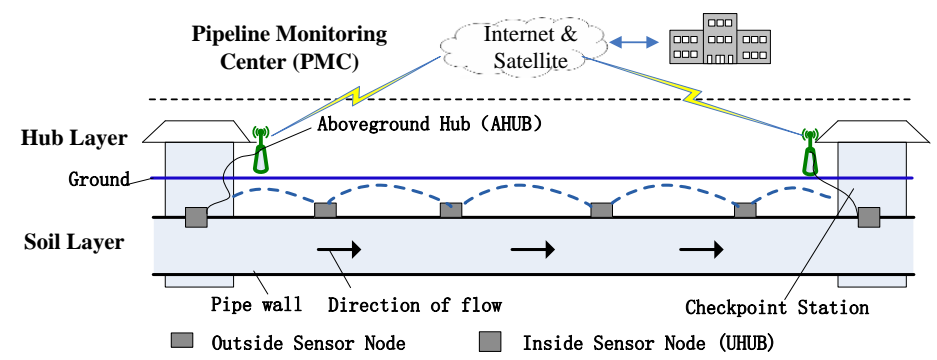

Figure 6. Three-tiers Network Architecture

In the soil layer, sensor nodes (that is outside sensor node) are deployed along the underground oil and gas pipeline surface to realize the seamless full coverage and connectivity for pipeline system. The outside sensor nodes can provide high granularity soil state information near the pipeline system in real time, such as soil temperature, soil moisture content, soil hydrocarbon contents, environmental pollution and weather information. Each outside sensor node has restricted computing and storage capability and limited battery, which cannot be recharged. Therefore, it is important that the outside sensor nodes deployment and scheduling must be energy-efficient so as to prolong system lifetime.

In the hub layer, sensor nodes are installed at initial injection station, compressor or pump station, partial delivery station, checkpoint station, valve station and final delivery station [2], which include the inside sensor nodes and aboveground 
gateway (HUB). The inside sensor nodes are only deployed inside the pipeline at the middle site of pipeline system to provide low granularity pipeline inside state information, such as pressure, fluid temperature, quantity of flow and pipeline leak, which connect with the aboveground sensor nodes directly. Each sensor node in the hub layer has abundant computing and storage resource compared to outside sensor nodes. Furthermore, these nodes can be recharged in time. The main functions of hub layer include analyzing data from outside sensor nodes and uploading the data to pipeline monitoring center (PMC).

PMC connects all sensor nodes in the hub layer by Internet or satellite communication. PMC is responsible for forming the overall analysis and issuing the information to managers. The operational procedure includes three steps [6]:

(1) In the first step, the inside sensor nodes uninterruptedly measure the flow pressure, fluid temperature and rate of flow in the pipe and send the measurements to the remote PMC day and night. Through the analysis model of the pipeline network based on the measurements, the PMC can identify some suspicious areas where the oil and gas pipelines are possible to have risk.

(2) In the second step, PMC activated the outside sensors in the suspicious areas to measure the required soil properties. The measurements are then sent to the sensor nodes in the hub layer through a multi-hop fashion. In addition, PMC can arrange the aboveground mobile node to the suspicious area to collect further information.

(3) In the thirdly step, the sensor nodes in the hub layer uploading oil and gas pipeline monitoring center accord the data collected in further to PMC. PMC make final decisions about the state of oil and gas pipelines.

\section{Improvement Approaches for Network Architecture}

In this section, we propose some improvement approaches based on the typical 3 tier deployment structure of WUSN for oil and gas pipeline monitoring and develop detail simulation analysis and discussion of the improvement strategies at same time.

\subsection{Sensor Nodes with Magnetic Induction Waveguide Coils}

WUSN for oil and gas pipeline monitoring are typical long linear topology structure, the longest transmission distance of sensor node is not more than $4 \mathrm{~m}$ and the dynamic change of channel conditions in soil is severe, which will lead the high energy consumption for long distance oil and gas pipeline monitoring. The magnetic induction (marked as MI, the path loss (denoted as LMI(d)) formula is given in (3)) and MI waveguide (marked as MIG, the path loss (denoted as LMIG(d)) formula is given in (4)) techniques can be developed to solve the problems based on EM wave techniques and provide more favorable advantages for WUSN in soil: the single hop communication range is greatly enlarged to $100 \mathrm{~m}$ and the MI channel conditions remain constant in soil since the attenuation rate of magnetic fields does not change in non-magnetic media [11], the experiment and simulation results are shown in Figure 7.

$$
\begin{aligned}
& L_{M I}(d) \cong 6.02+60 \log (d)+10 \log \frac{N_{t}}{N_{r} a_{t}^{3} a_{r}^{3}}, \\
& L_{M I G}(d) \cong 10 \log \frac{4\left(Z_{(n-1) n}+R\right)}{R}+20 \log \xi\left(\frac{R}{\omega M}, n-1\right),
\end{aligned}
$$


The three tiers network architecture with MIG communication module is shown in Figure 8. In the figure, each sensor node is equipped with a magnetic induction relay coil, which does not consume extra energy and unit cost can be neglected. And the coil is easy to deploy on the surface of pipeline and does not need extra maintenance because coil does not deviate from the ideal positions due to the pipeline structure.

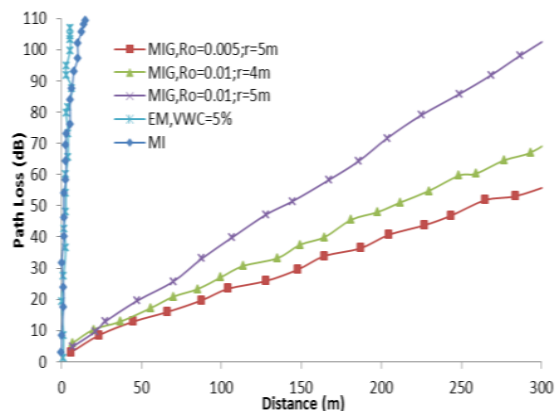

Figure 7. Path Loss Versus Internode
Distance based on EM, MI, MIG

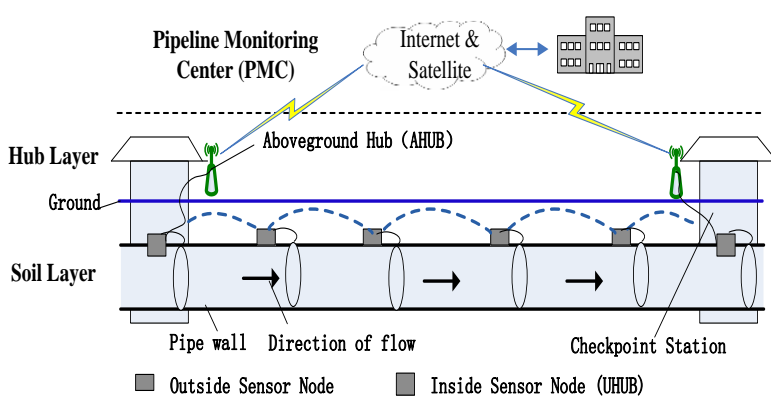

Figure 8. Three Tiers Network Architecture with Magnetic Induction Waveguide (MIG) Communication Module

To minimize the deployment cost while maintaining connection performance between two adjacent outside sensor nodes, the MIG should use the minimum number of relay coils to connect the two sensors on the link. According to (4) and proceeding reasonable hypothesis and simplification for parameters, the optimal number of relay coils for this link can be calculated as formula (5) and the numerical results are given in figure 9 [12]. It shows that the optimal number of the relay coils increases faster than the link length increases. Consequently, the required interval between two adjacent coils decreases as the link length increases.

$$
n_{\text {opt }}(d, B)=\arg \min _{n}\left\{P_{t}-L_{M I}\left(d, n, \omega_{0}+0.5 B\right) \geq \mathrm{P}_{\mathrm{th}}\right\},
$$

\subsection{Mobile Node Deployment Strategy}

In order to further reduce the energy consumption of underground sensor nodes, the hub layer can configure mobile nodes (include aboveground mobile nodes and underground mobile nodes) to move along oil and gas pipelines to collect information from underground sensor nodes (include outside sensor nodes and inside sensor nodes) or direct detection the pipeline state information based on the management requirements of oil and gas pipelines. It is obvious that mobile nodes can effectively improve the energy performance.

Aboveground mobile nodes

The aboveground mobile nodes can be carried by walking human, moving car, helicopter and unmanned aerial vehicle and so on. Three tiers network architecture with MIG communication module and aboveground mobile node (AM) is shown in figure 10 . 




Figure 9. Optimal Number of Relay Coils using MIG

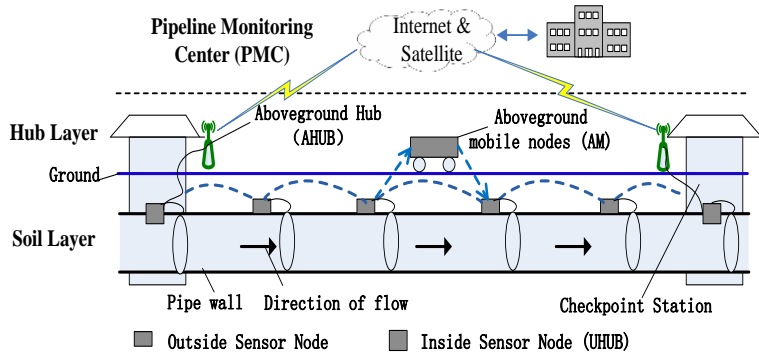

Figure 10. Three Tiers Network Architecture with MIG Communication Module and Aboveground Mobile Node (AM)

In Figure 10, besides the communication channel between underground (UG) sensor nodes (channel is UG-UG), the channels between aboveground (AG) mobile nodes and underground (UG) sensor nodes are the underground-to-aboveground (UG-AG) channel and the aboveground-to-underground (AG-UG) channel. The signal loss formulas of UG-UG, UG-AG and AG-UG are given in (1), (6) and (7) respectively [12]. The numerical results of the transmission ranges of the channels in WUSN are given in Figure 11. It shows that RUG-AG and RAG-UG are in the range of $10 \mathrm{~m}$ to $50 \mathrm{~m}$ and the RUG-AG is greater than RAG-UG.

$$
\begin{aligned}
& L_{U G-A G}=L_{U G}\left(d_{U G}\right)+L_{A G}\left(d_{A G}\right)+L_{U G_{-} A G}^{R}, \\
& L_{\mathrm{A} G-U G}=L_{U G}\left(d_{U G}\right)+L_{A G}\left(d_{A G}\right)+L_{\mathrm{A} G_{-} U G}^{R},
\end{aligned}
$$

Underground mobile nodes.

In some practical applications, only deploying the inside sensor nodes at the middle site of pipeline cannot effectively monitor the inside state of pipeline. The effective way to solve the problem is deploying inside sensor nodes in the entire inner of pipeline rather than only deployed at the middle site of pipeline system. But the pipelines are usually made from steel which is complete shielding for electromagnetic wave; therefore, inside sensor nodes cannot transmit the monitored data to HUB by aboveground mobile nodes, and only by multi-hops in inside sensor nodes which is not energy efficient data collection method obviously. We will take the underground mobile (UM) node mechanism to solve the above problems. UM node is moved by the flow of the fluid in pipeline along the pipeline to collect the monitored data from inside sensor nodes. Three tiers network architecture with MIG communication module and mobile node (UM and AM) is shown in figure 12.

The moving speed analysis of mobile node.

The moving speed of mobile node (v) has significant effect on average delay and data transfer success rate. In order to simplify the simulation analysis, we use one mobile node to collect data from underground sensor nodes (include inside sensor nodes and outside sensor nodes) directly and simulation result are shown in figure 13-14, in the figures, Len denotes the length of single data packet. It is obvious that choosing appropriate moving speed of mobile node is very important (the optimal velocity of mobile node is about $5-20 \mathrm{~m} / \mathrm{s}$ in figure $13-14$ ), low speed leads to the larger average delay and packet loss rate (data overflow memory of sensor node), but over quick speed similarly results in the increase of average delay and packet loss rate because that mobile node can't receive a whole packet during his one visit. Therefore, reducing the data packet split rate is very important for data collection performance. 




Figure 11. Transmission Ranges of the Three Channels in WUSN with Different Soil Water Content (SWC)

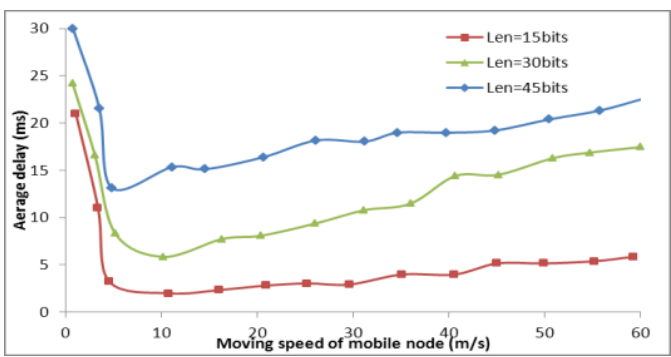

Figure 13. Moving Speed of Mobile Node and Packet Size Versus Average Delay

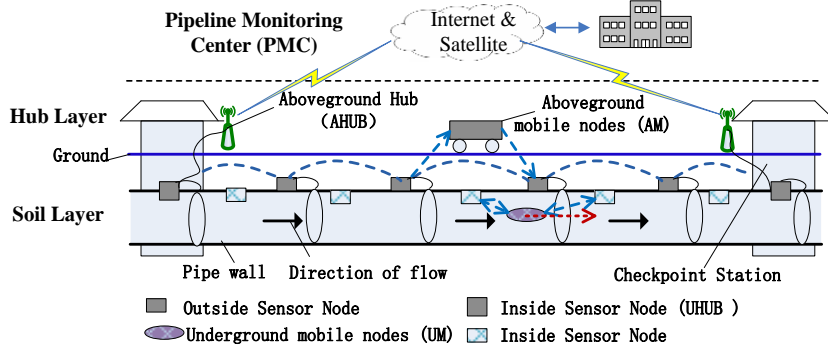

Figure 12. Three Tiers Network Architecture with MIG Communication Module, Underground Mobile Node (UM) and Aboveground Mobile Node (AM)

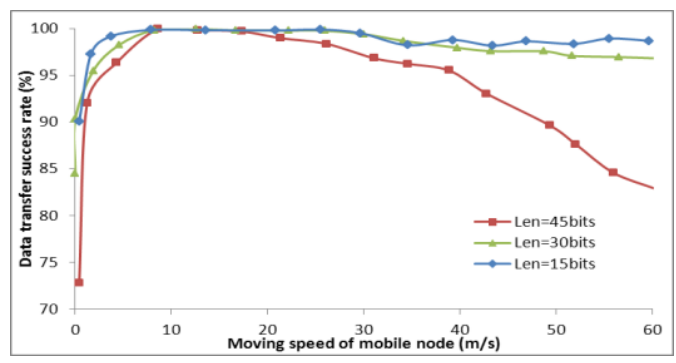

Figure 14. Moving Speed of Mobile Node and Packet Size Versus Data Transfer Success Rate

In order to clearly describe the data packet split problem and ensure that the data packet split does note happen in the process of data packet transmission, as shown in Figure 15, we define a zero data packet split area (shaded area), which is expressed with letter $\mathrm{H}$ which denotes the probability of zero split, namely underground sensor nodes and mobile nodes keep 100\% connection within time T. The boundary of the region $\mathrm{H}$ is made up of two cut circle with the radius Rc. Obviously, if $\mathrm{H}$ is regarded as the queue system, the greater the area of $\mathrm{H}$, the lower the average data packet split rate. And the area of zero data packet split area $\mathrm{H}$ is computed as formula (8).

$$
H_{\text {area }}=4 \times\left(\frac{R_{c}^{2}}{2} \arccos \sqrt{\frac{v T}{2 R_{c}}}-\frac{v T}{4} \sqrt{R_{c}^{2}-\left(\frac{v T}{2}\right)^{2}}\right), \quad r \geq \frac{v T}{2},
$$

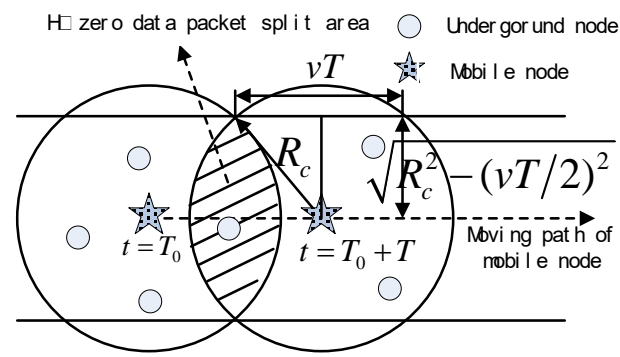

Figure 15. The Relationship among of Zero Data Packet Split Region $\mathrm{H}$ and $\mathrm{Rc}, \mathrm{v}, \mathrm{T}$

It is obvious that Harea increases monotonously with the radius Rc, therefore, we can expand Harea through increasing the transmission radius Rc of mobile node, reducing moving speed $\mathrm{v}$ of mobile node and temporal $\mathrm{T}$. 


\subsection{Using the Kinetic Energy of Oil and Gas Flow in Pipelines to Recharge Sensor Nodes}

Improving the transmission power of sensor node $(\mathrm{Pt})$ can effectively extend the transmission distance $(\mathrm{Rc})$. But the underground sensor node is powered by battery and its energy is extreme limited. Compared to that in air, the energy consumption is much more. Furthermore, the underground environment also not suitable for installing solar energy charging devices compared to the aboveground environment. According to the specific application environment of underground oil and gas pipeline, we designed a new type of sensor nodes, which is particularly suitable for underground oil and gas pipeline monitoring. And the sensor node uses the kinetic energy of oil and gas flow in pipelines to recharge. The structure diagram of the sensor node is shown in Figure 16.

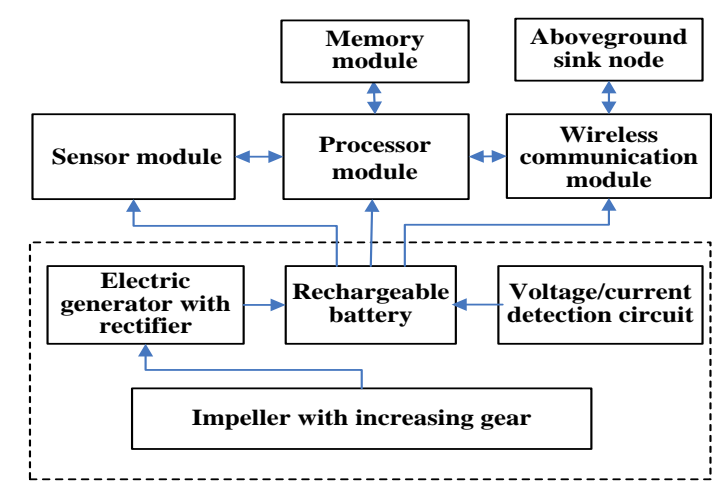

\section{Figure 16. Structure Diagram of Sensor Node that use the Kinetic Energy of the Fluid in Pipelines to Charge}

In Figure 16, the impeller was firstly drove by kinetic energy of oil and gas flow in the oil and gas pipelines, and then the impeller drove the electric generator to charge the rechargeable batteries. In this way, the energy supply of sensor nodes can be guaranteed, which will effectively prolong the network life cycle. At the same time, this technique also lay a good foundation for improving the transmission power of sensor nodes, extending the single hop transmission distance of the sensor nodes and promoting the connectivity of network. The three tiers network architecture with MIG communication module, charging unit and mobile node (UM and $\mathrm{AM}$ ) is shown in Figure 17.

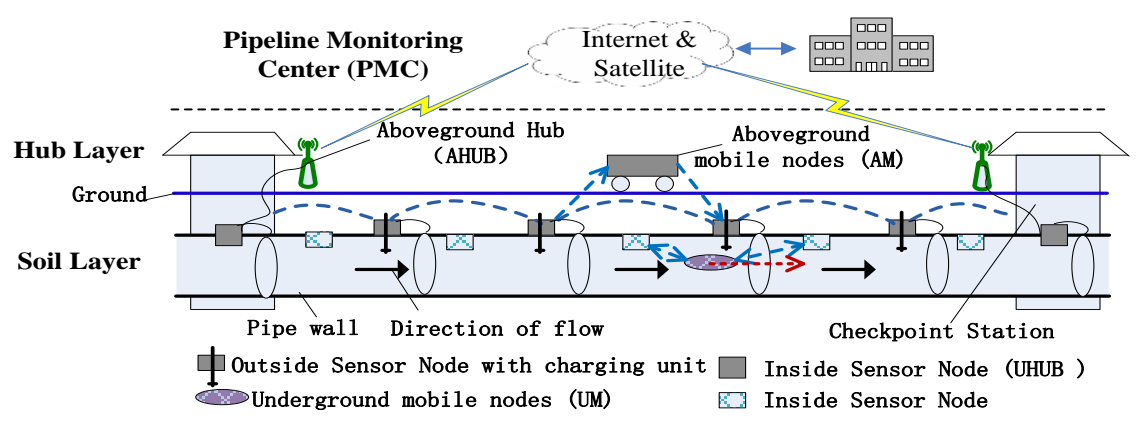

Figure 17. Three Tiers Network Architecture with MIG Communication Module, Charging Unit, Underground Mobile Node (UM) and Aboveground Mobile Node (AM) 


\subsection{Sensor Nodes Line Deployment and Scheduling Strategies}

The WUSN for oil and gas pipeline monitoring is typical linear topology. In this linear network topology, the vast majority of sensor nodes cannot directly communicate with the gathering node (such as the inside sensor nodes and aboveground mobile nodes in the hub layer), which leads the energy consumption of sensor nodes near the gathering nodes to be consumed quickly and then soon to "death". The "death" of sensor nodes is fatal for the whole network because the data of other sensor nodes can't be sent to the gathering nodes by means of the relay way. There are two types of sensor nodes deployment and scheduling strategies which are designed and analyzed as follows.

Non-uniform deployment and scheduling strategy

In order to avoid unbalanced energy consumption in underground sensor nodes (USN, which includes inside and outside sensor node), we use the non-uniform line deployment strategy [13] to prolong the life span of network. The deployment strategy is as shown in Figure 18.

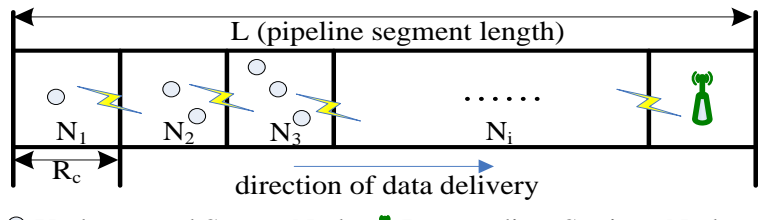

Underground Sensor Node Intermediate Stations Nodes

\section{Figure 18. Non-uniform Deployment and Scheduling Strategy for Energy Consumption Balance in Long Line Structure}

In Figure 18, L denotes the distance of multi-hop transmission with USNs, Rc denotes communication radius of USN, and the pipeline segment is divided into $n$ $(n=[L / R c])$ sections. Ni denotes the number of USNs in the $i$-th section $(i \in[1, n])$. In order to keep the ratios same of the number of energy consumption in each section to the total amount of energy in the section, we should deploy more USNs in the sections closer to HUB, and deploy lesser USNs in the sections far from HUB. We have:

$$
N_{1}<N_{2}<N_{3}<\cdots<N_{i}<\cdots<N_{n},
$$

Furthermore, the Ni is computed as follows:

$$
N_{1} / E_{1}=N_{2} / E_{2}=\cdots=N_{i} / E_{i}=\cdots=N_{n} / E_{n},
$$

In (10), Ei denotes the total energy consumption of USNs in the i-th section and the value of Ei depends on data packet generating rate. Moreover, we can further prolong the life span of network by scheduling USNs to active state and sleeping state. Each section has only one active USN to sense and relay data packet to HUB in the same time. The probability when USN is active in the $\mathrm{i}$-th section is:

$$
p(i)=1 / N_{i} \text {, }
$$

Uniform deployment and scheduling strategy

When USNs are deployed in the uniform way, non-uniform partition strategy [5] is very necessary to introduce to balance the network energy consumption, which is shown in figure 19. In the figure, the monitoring area (oil and gas pipeline) is divided into $\mathrm{M}$ subareas marked as A1, A2,...,AM, respectively. The length of each subarea on the $\mathrm{Z}$ axis is L1, L2,...LM. The average communication distance (di) of adjacent partitions of $\mathrm{Ai}$ and $\mathrm{Ai}+1$ is $\mathrm{di}=(\mathrm{Li}+\mathrm{Li}+1) / 2$ on the direction of $\mathrm{Z}$ axis. 




\section{Figure 19. Uniform Deployment and Scheduling Strategy for Energy Consumption Balance in Long Line Structure}

Supposing that events to be tested in the monitoring area will occur with the probability of uniform distribution within unit time, and the amount of data collection of each sensor node is $\mathrm{k}$. the energy dissipations (Ei) of the sensor node $\mathrm{i}$ ( $\mathrm{Si}$ ) include the energy consumption of receiving the data from the $\mathrm{Si}-1$, the energy consumption of forwarding the data from the $\mathrm{Si}-1$ and the energy consumption of sending the data of the $\mathrm{Si}$ within unit time, the computational formula can be developed as (12) based on (2).

$$
E_{i}=\left(E_{\text {dec }} \times N \times k \times \frac{\sum_{j=1}^{i-1} L_{j}}{L}\right)+\left(E_{\text {dec }} \times N \times k \times \frac{\sum_{j=1}^{i} L_{j}}{L}+\varepsilon_{\text {amp }} \times N \times k \times \frac{\sum_{j=1}^{i} L_{j}}{L}\left(d_{i}^{\gamma}\right)\right),
$$

In (12), $\mathrm{N}$ is the total number of outside sensor nodes deployed in pipeline system. Furthermore, we can have $\mathrm{Ei}=\mathrm{Ei}+1$ because the energy consumption of each subarea (Ai) need to be balanced to prolong the network lifetime. Then the (12) can be expressed as,

$$
L_{i+2}=2 \times\left(\frac{\sum_{j=1}^{i} L_{j} \times\left(\frac{L_{i}+L_{i+1}}{2}\right)^{\gamma}-\frac{E_{\text {elec }} \times\left(L_{i}+L_{i+1}\right.}{\varepsilon_{\text {anp }}}}{\sum_{j=1}^{i+1} L_{j}}\right)^{\frac{1}{\gamma}}-L_{i+1},
$$

In (13), the value of L1 should be as large as possible to ensure that time delay of network is as little as possible, and $\mathrm{Li}>\mathrm{Li}+1$. In order to ensure that subarea within the scope of the effective communication of sensor node, there is $\mathrm{L} 1+\mathrm{L} 2<=\mathrm{Rc}$. and then we can get the length of each subarea (Li).

After partition, the lifetime of every subarea is the same, so the lifetime of the partition A1 is network lifetime. Assume that the initial energy of each sensor node is Einit and the total energy of entire network is Einit $\times N$. Since the sensor node is uniform deployment, therefore, the total energy of subarea A1 is in the EA1= Einit $\times \mathrm{N} \times \mathrm{L} 1 / \mathrm{L}$, the network life span can be calculated as,

$$
T=\frac{E_{\text {A1 }}}{E_{i}}=\frac{E_{\text {init }}}{k \times\left(E_{\text {elec }}+\varepsilon_{\text {amp }} \times\left(\left(L_{1}+L_{2}\right) / 2\right)^{\gamma}\right)},
$$

\section{Summary}

In this work, we carefully analyzed the underground wireless channel characteristics of near-surface underground soil environment with electro magic wave, energy consumption model and application requirement of WUSN for oil and gas monitoring, and presented the typical three layer architecture of WUSN. In order to further improve the energy efficient and energy balance of the network performance, we put forward four strategies and develop detailed simulation analysis. The analysis results show that these strategies can effectively increase energy performance of network. In future, we plan to further investigate sensor node scheduling technologies based on our improved three tires network architecture and the data fusion and prediction to further enhance network lifespan. 


\section{Acknowledgments}

The authors are grateful to the anonymous referees for their valuable comments and suggestions. The research is supported by the Hubei province science and technology research plan key project (Grant No. D20161304) and National Natural Science Foundation of China (Grant No. 41372155).

\section{References}

[1]. I. F. Akyildiz; E. P. Stuntebeek. Wireless underground sensor networks: research challenges.// Ad Hoc Networks, Vol.4, (2006), pp.669-686.

[2]. Y

$$
\text { Wikipedia. }
$$

Pipeline

transport.

URL: http://en.wikipedia.org/wiki/Oil_pipeline\#For_oil_or_natural_gas.(08.22.2014).

[3]. H.P. Yu; M. Guo. An efficient oil and gas pipeline monitoring systems based on wireless sensor networks.// in Proceedings of the Information Security and Intelligent Control/ Taiwan, China, 11-13 August (2012), pp.188-193.

[4]. Ivan Stoianov; Lama Nachman; and Sam Madden. PIPENET: A Wireless Sensor Network for Pipeline Monitoring.// Proceedings of the 2007 IPSN./ Cambridge, Massachusetts, U.S.A, (2007).

[5]. Hua-ping Yu; Hang Qin; Jian Wang; and Mei Guo. Deployment algorithms of wireless sensor networks for near-surface underground oil and gas pipeline monitoring.// Sensors \& Transducers, Vol.174, Issue 7, (2014), pp.95-102.

[6]. Z. Sun; P. Wang; M.C. Vuran; M. A. Al-Rodhaan; A. M. Al-Dhelaan; I. F. Akyildiz, MISEPIPE: Magnetic induction-based wireless sensor networks for underground pipeline monitoring.// Ad Hoc Networks, Vol.9, (2011), pp.218-227.

[7]. M. C.Vuran; I.F.Akyildiz. Channel model and analysis for wireless underground sensor networks in soil medium.// Physical Communication,Vol.3, (2010),pp.245-254.

[8]. Heinzelman W B; Chandrakasan A P; Balakrishnan H. An application-specific protocol architecture for wireless microsensor networks.// IEEE Transactions on wireless communications, (2002), 1(4), 660-670.

[9]. L. Li; Y.A. Liu. Sensor deployment algorithm for wireless underground sensor network.// Journal of Jilin University (Information Science Edition), Vol.25, Issue 6, (2007), pp.15-20.

[10]. H. P. Yu; C. X. Wu; M. Guo. Energy efficiency analysis for mobile wireless sensor network.// Computer Engineering, Vol.35, Issue 14, (2009), pp.127-129.

[11].Z. Sun; I. F. Akyildiz. Magnetic inuction cmmunications for wireless underground sensor networks.// IEEE Transactions on Antenna and Propagation, Vol. 58, , Issue 7, (2010), pp.24262435.

[12]. Z. Sun. Reliable and efficient communication in wireless underground sensor networks.// Doctor of Philosophy in the school of electrical and computer engineering, Georgia Institute of Technology, August (2011).

[13]. Yuan Hui-yong; Zhao Xiao-lian; and Xie Dong. Node deployment strategy in line wireless sensor networks.// The journal of computer Engineering and Applications, vol.46, no.7, (2010), pp.86-88.

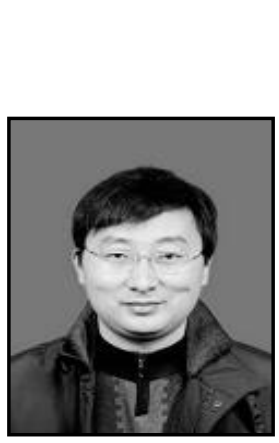

\section{Authors}

Huaping Yu, received his M.S. and Ph.D. degrees in Computer Engineering from the Yangtze University, Jingzhou, China, in 2008 and 2015, respectively.

He is currently a researcher in the oil and gas pipeline monitoring and wireless sensor network laboratory. His current research interests include oil and gas field production safety monitoring and wireless sensor networks.

He is associate professor of College of Computer Science, Yangtze University. Recently, he has taken 15 research projects at all levels, and has more than 40 academic theses being published. Currently, he is the member of China computer federation. 




Lan Huang, received her Ph.D. in Computer Science from the University of Waikato, New Zealand, in 2011, and B.S. and M.S. degrees in Information Sciences from Beijing Normal University, China, in 2003 and 2006, respectively. She is a lecturer of the Computer Science department at Yangtze University. Her research interests include data mining and text analysis.

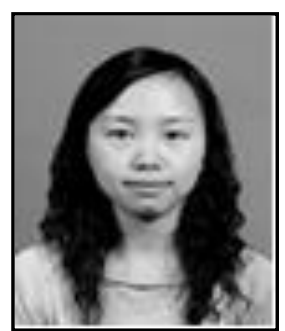

Mei Guo, received her B.S. and M.S. degrees from the Southwest China Normal University, Central China Normal University, in 2003 and 2009, respectively. She is currently a research assistant in the oil and gas pipeline monitoring and wireless sensor network laboratory. Her current research interests include oil and gas field production safety monitoring and wireless sensor networks. 